\title{
Application of Motion Sensor Based on Neural Network in Basketball Technology and Physical Fitness Evaluation System
}

\author{
Bin Yuan, ${ }^{1}$ M. M. Kamruzzaman $\left(\mathbb{D}^{2},{ }^{2}\right.$ and Shaonan Shan $(\mathbb{D})^{3,4}$ \\ ${ }^{1}$ School of Physical Education, Chengdu Normal University, Chengdu, 611130 Sichuan, China \\ ${ }^{2}$ Department of Computer and Information Science, Jouf University, Sakaka, Al Jouf 72311, Saudi Arabia \\ ${ }^{3}$ School of Urban Economics and Public Administration, Capital University of Economics and Business, Beijing 100070, China \\ ${ }^{4}$ School of Business Management, Liaoning Vocation Technical College of Modern Service, Shenyang, 110000 Liaoning, China \\ Correspondence should be addressed to Shaonan Shan; rose_1844100@cueb.edu.cn
}

Received 25 January 2021; Revised 26 February 2021; Accepted 31 March 2021; Published 15 April 2021

Academic Editor: Wenqing Wu

Copyright ( 2021 Bin Yuan et al. This is an open access article distributed under the Creative Commons Attribution License, which permits unrestricted use, distribution, and reproduction in any medium, provided the original work is properly cited.

\begin{abstract}
Basketball is a sport that requires high athletes' skills and physical fitness and is deeply loved by the people in our country. This paper studies the application of neural network-based motion sensors in basketball technology and physical fitness evaluation system. The ideal effect of the system is to scientifically analyze relevant data through intelligent algorithms and provide more accurate diagnosis suggestions. Recognizing human movements requires collecting various data of the human body through motion sensors. The data acquisition components of this system are based on considerations of portability and power consumption and are equipped with equipment with strong computing power to realize the functions of data preprocessing, training, and recognition of the recognition model. The system only needs to send the data in the data collector to the computing device; it can effectively realize the action recognition and judge whether the athlete's technical action and physical fitness level meet the standard. From the experimental data, the pass rate of the subjects in the 1000 -meter run was $83.3 \%$, and the excellent rate was $10 \%$; the pass rate in the 1 -mile run was $90 \%$, and the excellent rate was $6.7 \%$; and the pass rate in the 20 meter round trip was only at $56.67 \%$; it can be seen that there is still room for improvement in the reaction speed and agility of most subjects. According to intelligent data analysis, athletes can better understand where they have shortcomings and improve their physical fitness and basketball skills through targeted training.
\end{abstract}

\section{Introduction}

With the advancement of science and technology, intelligent systems have been rapidly developed in various fields, and the availability and use speed of wearable sensor technology have also grown rapidly. The aspects that need to be considered when acquiring athlete information outside the laboratory environment are severely affected and dependent on currently available technologies. Through technological innovation and development, new sensor equipment and systems will provide the possibility for them. Wearable sensor technology is gradually popular in the sports field, which can provide data safely and effectively, promote the development of sports, and meet the needs of coaches and athletes for equipment. Basketball is a sport that requires a constantly changing rhythm, requiring speed, acceleration, and explo- sive power, such as rebounds, layups, jump shots, and fast breaks. There is a big gap between our country's basketball skills and the United States and European basketball powers. Therefore, it is particularly important to strive to improve the technical and physical level of basketball players and to train excellent basketball players.

The application of mobile sensors in various fields in foreign countries started early, and many good results have been achieved in the research of intelligent sports systems. Conte conducted research on the physical and technical requirements of young basketball players' training. In order to examine the physiological and technical requirements of No Game Training (NDGD) and Conventional Training (RD), he tested 19 young basketball players and used Wilcoxon symbols to test and evaluate the relationship between NDGD and RD conditions for each dependent variable. 
From the experimental results, this research has played a role in optimizing the training program of young athletes, but due to the small number of research objects, it has affected the credibility of the final conclusion [1]. Santos conducted an in-depth study on the changing laws of basketball technical indicators. He conducted a survey on the data of the Brazilian basketball championship from 2005 to 2015 and analyzed the changes in 13 standard indicators in the basketball game. For basketball players, good physical fitness and accurate grasp of technical standards are essential. If the basketball technical indicators and physical fitness evaluation standards can be analyzed through the intelligent system, the scientific nature of basketball training can be further improved [2].

Although domestic research on artificial neural networks and sports intelligence started relatively late, they have developed rapidly. Yuzhou carried out research on basketball multidirectional training and technical analysis based on $\mathrm{BP}$ neural network model. Artificial neural networks can acquire various abilities through learning. If the characteristics of the brain neural network can be used reasonably, an intelligent system with similar functions to the human brain can be designed to process various information. He and his team analyzed the multidirectional training and technical analysis of basketball based on the BP neural network model. Through the analysis of the data, the basketball players' training and team strategy are optimized. Taking the smallest error and the highest accuracy as the standard, the best method was selected as the predictive model for each performance. From the experimental test results, the model can be helpful to improve basketball training, but more experimental data is needed to improve the accuracy of the model [3].

Based on artificial neural networks and mobile smart sensors, this paper conducts in-depth research on physical fitness assessment and technical improvement training in basketball training. The research is mainly carried out from the following aspects. This article introduces the technologies and methods involved in the basketball training system, including neural network-based mobile sensors, decision tree classification algorithms, wavelet transform, and system data acquisition technology. Based on the analysis of product requirements, this article has completed the development of the intelligent basketball training system and introduced the overall structure and data modules of the system in detail. Finally, this article starts from the two directions of basketball technical statistical indicators and physical fitness evaluation, combined with experimental data to verify the positive impact of the system in improving the effect of basketball training.

\section{Application of Technology in Basketball Test and Evaluation System Based on Neural Network}

2.1. Mobile Sensor Based on Neural Network. Artificial neural network is a technology that simulates the neurons of the human brain for abstract processing and realizes information processing by establishing models [4]. With the continuous deepening of neural network research, it has become more and more widely used in the fields of intelligent machines, intelligent recognition, intelligent prediction, and automatic control. Real-time recognition of human body data based on neural networks using mobile sensors can provide data support for intelligent evaluation systems to make scientific decisions $[5,6]$.

Radio frequency identification technology (RFID) is a kind of communication technology, also called radio frequency identification or electronic tag [7]. It recognizes specific targets and reads data mainly through radio signals. Commonly used technologies mainly include passive, low frequency, ultrahigh frequency, and high frequency. This technology is mainly used for data function positioning in basketball training [8]. The sensor is a kind of signal conversion device, which is mainly composed of a conversion original and a sensitive element. The sensor has the function of sensing external signals and detecting, including heat, light, and humidity, and at the same time transmitting the acquired signals to other organs and devices [9].

2.2. Decision Tree Classification Algorithm. The human motion data collected by sensors is based on the reference system of each sensor [10]. Since human movement cannot be described in accordance with the sensor's reference system, such source data cannot be used directly, so it is necessary to classify and process the data through related technologies. Decision tree algorithm is a common machine learning algorithm. When doing machine learning, the decision tree uses a tree structure to resolve the problem one by one [11]. Decision trees make selection judgments at each node, which is similar to the process of human selection when facing some decision problems [12].

Information entropy, information gain, conditional entropy, and information gain ratio are commonly used in decision trees as the basis for division. Information entropy indicates whether the sample subset is single, that is, the smaller the value, the single the type of data in the set, which means that the division is more ideal [13]. Information entropy is defined as

$$
\operatorname{Eet}(D)=-\sum_{k=1}^{|v|} p k \log 2 p k
$$

Assuming that there are $V$ possible values for the discrete attribute $a$, the information gain can be calculated according to the weights assigned to the branch nodes according to the number of samples:

$$
\operatorname{Gain}(D, a)=\operatorname{Ent}(D)-\sum_{v=1}^{V} \frac{\left|D^{V}\right|}{|D|} \operatorname{Ent}\left(D^{V}\right)
$$

According to the definition of information gain, attributes that account for the majority of numbers have inherent advantages and therefore make the division effect worse. The decision tree algorithm is different from the above; it uses the gain rate as the partition index to minimize the impact [14]. Its definition is 


$$
\operatorname{Gain} \_ \text {ratio }(D, a)=\frac{\operatorname{Gain}(D, a)}{I V(a)} .
$$

Among them

$$
I V(a)=-\sum_{v=1}^{V} \frac{\left|D^{V}\right|}{|D|} \log 2 \frac{\left|D^{V}\right|}{|D|} .
$$

This is called the "intrinsic value" of the attribute. At the same time, the decision tree is divided by the Gini index. The Gini value can represent the unity of data, and its definition satisfies

$$
\begin{aligned}
\operatorname{Gini} \_\operatorname{index}(D, a) & =\sum_{v=1}^{V} \frac{\left|D^{v}\right|}{|D|} \operatorname{Gini}\left(D^{V}\right), \\
\operatorname{Gini}(D, a) & =\sum_{k=1}^{|v|} \sum_{k \neq k} p k p k=1-\sum_{k=1}^{|y|} p_{k}^{2} .
\end{aligned}
$$

When the decision tree is divided, it will not only encounter discrete values but also divide continuous values. When conducting experiments, sampling is often used to discretize continuous values for continuous variables. For continuous variables, discrete values are essentially processed [15]. Therefore, dealing with the division of continuous variables is essentially dealing with the problem of a large number of possible discrete values of attributes. As samples are sampled, there will be some missing attributes in some samples. If only the samples with values on these attributes are divided, it will lead to a waste of sample sets.

Set each sample $x$ to be given a weight $w x$ and define

$$
\begin{aligned}
\rho & =\frac{\sum x \in \tilde{D}^{W x}}{\sum x \in D^{W x}}, \\
\tilde{P} k & =\frac{\sum x \in D k^{W x}}{\sum x \in \tilde{D}^{W x}}(1 \leq k \leq|y|), \\
\tilde{r} k & =\frac{\sum x \in \tilde{D} v^{W x}}{\sum x \in \tilde{D}^{W x}}(1 \leq v \leq|V|) .
\end{aligned}
$$

According to formulas (7)-(9), the promotion of information gain can be defined to satisfy

$$
\begin{aligned}
\operatorname{Gain}(D, a) & =\rho \times \operatorname{Gain}(\tilde{D}, a) \\
& =\rho \times\left(\operatorname{Ent}(\tilde{D})-\sum_{v=1}^{V} \tilde{r} v \operatorname{Ent}\left(\tilde{D}^{v}\right)\right), \\
& \operatorname{Ent}(\tilde{D})=-\sum_{k=1}^{|y|} \tilde{p} k \log 2 \tilde{p} k .
\end{aligned}
$$

Since the classification boundary formed by the decision tree is characterized by being parallel to the coordinate axis, when the boundary of the attribute value to be classified is more complicated, the decision tree must be divided into multiple segments due to the axis-parallel attribute to achieve a good fitting effect. It will increase the number of decision tree layers, which will undoubtedly lead to increased training and prediction costs [16]. Therefore, if the function combination value of multiple attributes can be used for division, it will not only be parallel to the coordinate axis in the multidimensional division plane, which is to find a suitable linear classifier for the node.

2.3. Wavelet Transform. When identifying the specific pattern of data, it is necessary to understand the detailed characteristics of the data, such as the order and quantity of various frequencies, in order to better extract the signal characteristics and construct the recognition model. It is necessary to conduct a comprehensive time-frequency analysis of the signal, so it is necessary to introduce wavelet analysis [17].

Wavelet analysis decomposes the signal through wavelet transform and analyzes the signal according to the prominent local features of the signal by analyzing the local time domain or frequency domain. This solves the problem that the traditional Fourier transform cannot be analyzed from the global shortcomings of capturing the detailed characteristics of the signal, while solving the problem that the signal cannot be adaptively changed with the change of the signal after the fixed window size decomposition [18].

Let the expression of a basic function be $\psi(t)$, and let

$$
\psi a b(t)=\frac{1}{\sqrt{a}} \psi\left(\frac{t-b}{a}\right) .
$$

Among them, $a, b$ are constants, and $\psi a b(t)$ is the basic function obtained through translation and expansion. Given a square-integrable signal $x(t) \in L^{2}(R)$, the wavelet transform of $x(t)$ is defined as

$$
\operatorname{ETx}(a, b)=\frac{1}{\sqrt{a}} \int_{-\infty}^{+\infty} x(t) \psi^{*}\left(\frac{t-b}{a}\right) d t .
$$

Among them, $a, b$ represents the scale factor and time shift, respectively, and $\psi(t)$ is the basic wavelet. Let the Fourier transform of $x(t)$ be $X(\Omega)$, and then, the frequency domain expression of wavelet transform is

$$
W T x(a, b)=\frac{1}{2 \pi}(X(\Omega), \psi a, b(\Omega)) .
$$

A low-pass filter can filter out high-frequency noise to achieve denoising of human body motion data. However, this method cannot filter out the sensor jitter noise that is similar to the human body motion frequency, because the human body motion frequency is not fixed at a certain low frequency, but has a different frequency in each motion [19]. Figure 1 shows the steps of wavelet threshold denoising.

It can be seen from Figure 1 that the basic steps are as follows: wavelet transforms the signal, uses the threshold function to process each layer of wavelet separately, and gets the signal after wavelet reconstruction to remove noise. In the denoising process, by using the threshold function, the noise 


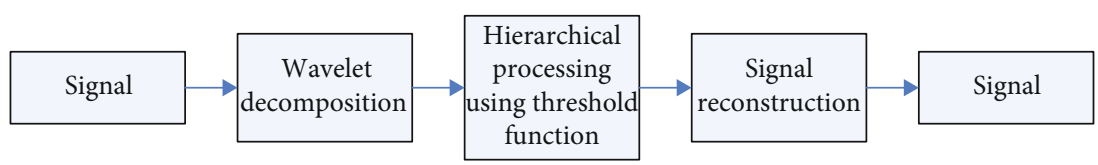

Figure 1: Wavelet threshold denoising steps.

below the threshold can be effectively filtered out [20]. If the selected threshold is too large, part of the effective information of the signal will be filtered out, resulting in serious signal distortion; if the selected threshold is too small, the denoising will be incomplete. Therefore, the choice of the threshold is related to the quality of the filtering effect.

For the joint distribution of multidimensional independent normal random variables, when the data tends to infinite dimensionality, the optimal threshold is calculated according to the maximum and minimum limits of the estimated data [21]. The constraints are

$$
\lambda=\sigma_{k} \sqrt{2 \ln k}
$$

where $\sigma_{k}$ represents the standard deviation of noise and $k$ represents the signal length. $\omega_{i}$ represents the square value of the wavelet coefficient, and assuming that the risk vector is $\vec{R}$, the element $r_{i}$ in it satisfies

$$
r_{i}=\frac{\left[n-2 i-(n-i) \omega_{i}+\sum_{k=1}^{i} \omega_{k}\right]}{n}, \quad i=1,2, \cdots, n .
$$

Find the minimum value from $r_{i}$ as the risk value, and then, the corresponding $\omega_{\min }$ can be obtained according to the above formula.

$$
\lambda=\sigma_{k} \sqrt{\omega_{\min }} .
$$

The basic idea of the extreme value criterion is to estimate the threshold value by producing the extreme value of the smallest mean square error. The threshold estimation formula is

$$
\sigma_{k}=\frac{\operatorname{mid}\left(W_{1}, k, 0 \leq k \leq 2^{j-1}-1\right)}{0.6745} .
$$

On the whole, different threshold estimation methods have different denoising effects. Therefore, in actual application, it is necessary to consider the thresholds obtained by different threshold estimation methods to compare the signal denoising effects [22].

2.4. System Data Acquisition Technology. The collection of basketball technology and data belongs to the process of automatically collecting information, and the sources of data collection are mainly other equipment under test and sensors [23]. The system has user-defined and flexible functions, with 8 slots and 4 slots as commonly used, but based on sensors and technical physical fitness tests, there are obstacles in data fusion, and further development is needed to realize the com- bination of network computers and sensors. To ensure the feasibility and accuracy of signal detection and then form a complete detection system [24], in this system, the sensor is a device that outputs signals and senses the measured size. Data is the main transmission content. When the above functional systems exist as independent individuals, the data transmission must be transmitted step by step. The data processing is mainly the conversion processing of the sensor output signal. The measurement results are displayed in analog or digital and recorded by an automatic recording device [25].

\section{Simulation Experiment of Basketball Technique and Physical Fitness Evaluation System Based on Neural Network}

3.1. Experimental Background. The country has always attached great importance to the improvement of the overall quality of the masses, hoping to promote the overall development of the masses' moral, intellectual, and physical capabilities. However, due to the impact of exam-oriented education, the lack of physical education teachers in grassroots schools has resulted in relatively low quality of physical education in grassroots schools. Basketball is one of the most popular sports in our country, but even professional basketball players cannot always keep one-on-one accompany training when they are training. At this stage, our country has also done a lot of research on basketball training, mainly from the perspective of analyzing common training problems and changing classroom teaching methods. However, the current problems in various physical education have not been well resolved. To this end, this article will use neural network-based mobile sensors to try to combine basketball technical training and physical fitness assessment with an intelligent system to improve the efficiency of basketball teaching. Participants in this experiment include 3 senior basketball coaches, 3 professional basketball players, and 30 basketball enthusiasts with varying technical levels. In this article, 30 subjects were divided into 6 groups (5 people in each group) of similar levels, and they were asked to wear mobile sensors to complete the required basketball technical action and physical fitness assessment items.

3.2. Experimental System Structure Design. Before proceeding with the system structure design, this article conducted a research and analysis of the basic functions of the product and determined that the main task of the system is to improve the scientific nature of the athlete's physical fitness assessment and provide targeted training and guidance programs for athletes to improve their basketball skills. With a detailed understanding and comprehensive analysis of the 


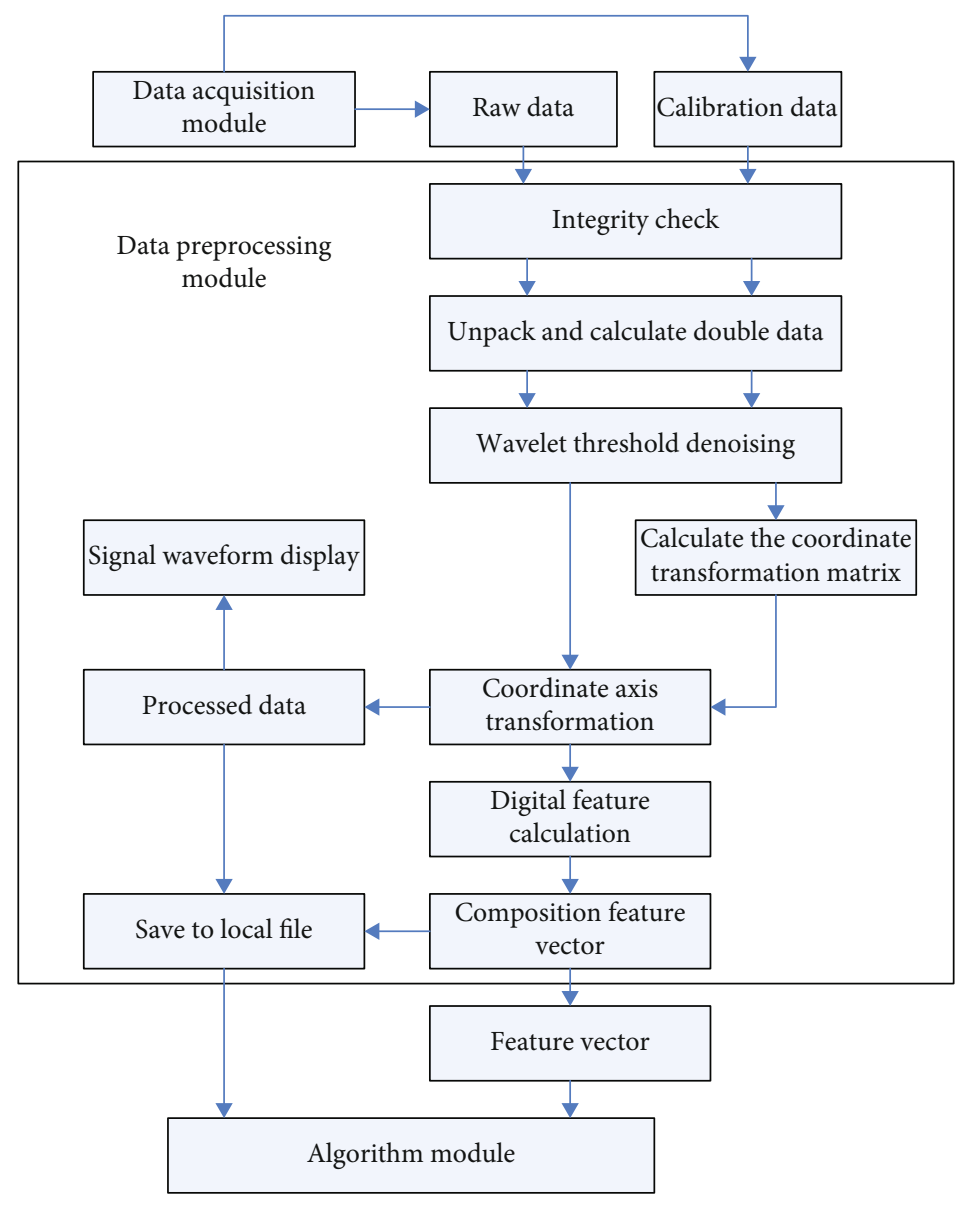

Figure 2: System data preprocessing process.

physical fitness characteristics and competition rules of basketball sports, the evaluation system of basketball physical fitness and technology is constructed by modern technology.

For intelligent systems, a reliable source of information and data is the basic prerequisite for intelligent analysis. When an athlete wears a smart device with a built-in mobile sensor, the instrument can detect the athlete anytime and anywhere and it can also achieve rapid multiparameter detection and feedback in terms of test data. The intelligent system uses a microcomputer as the data processing software monitoring processor, which can effectively complete the conversion of electronic signals to digital signals and has the characteristics of automation in scoring processing. The data analysis software also has the characteristics of networking and intelligence. It is the main reference basis for the analysis of technical actions.

The system mainly includes the following components: data analysis and performance recording software, basketball technical index database, and basketball special tester. The selected core control device is a single-chip microprocessor, the processing and analysis modes mainly include B/S mode and $\mathrm{C} / \mathrm{S}$ mode, and the front end of collecting intelligent data mainly includes a single-chip microprocessor, sensor, and corresponding interface circuit.
3.3. Data Module Design of Experimental System. The overall system is divided into three parts, namely, the data acquisition part, the data transmission part, and the data processing part. The data processing part to complete the functions including data preprocessing, training, and recognition of the recognition model is the key to the entire system. Considering that if an embedded device is used for algorithm implementation, it is necessary to select the device and the system architecture for this problem. The overall implementation cost and complexity are relatively high. At the same time, the PC also has certain portability. At the same time, it has $\mathrm{PC}$ equipment and development cost. And the complexity is low, so the PC is used to implement the algorithm here. Figure 2 shows the system data preprocessing process.

It can be seen from Figure 2 that the process of data preprocessing is as follows: firstly, pass data verification to determine the correctness of the data packet, then parse the data packet to obtain the decimal value of the original data, and finally filter the signal. After filtering the data, the data is transformed with coordinate axes to obtain the coordinates of the human body travel reference system. Finally, the digital features are calculated for these coordinates, and finally, the feature vector is obtained, and the feature vector is given to the algorithm module as input. 


\section{Motion Sensor Based on Neural Network in Basketball Technology and Physical Fitness Evaluation System}

\subsection{Motion Sensor Based on Neural Network in Improving} Basketball Technical Training. Basketball technical statistics is one of the methods used by sports researchers to organize, analyze, and research basketball game data. It has been widely used in basketball games today. It can intuitively and scientifically analyze some basketball game-related data. Coaches use data analysis to better study its own advantages and disadvantages, in order to finally win the game, help the team to formulate reasonable countermeasures, and implement the formulation plan; and the postgame summary needs to combine data statistics, field performance, etc. to make the game summary reach more accurate and targeted purpose. It is helpful to summarize the reasons for the failure and find out the winning method and avoid the same mistakes in the next game; through the accumulation and dataization of basketball game statistics, the establishment of a game database for the team and players can better understand the growth of the team and players to understand the player's training effect and the completion of tactics; at the same time, it also sets the task of playing technical and tactical indicators for the basketball team and players.

The only way to score in a basketball game is through the technical action of shooting, so the technical action of shooting is the most critical in basketball technology. The field goal percentage is an important indicator for evaluating the offensive team's shots. It is classified as two-pointers, threepointers, and free throws. The calculation formula is field goal percentage $=$ total field goal attempts/full field goal attempts. If the scoring is only supported by the number of shots, then the total field goal percentage does not reflect the shooting efficiency, and the coach may lose the right to know the team's offensive effect and affect the next tactical arrangement. Table 1 and Figure 3 are the statistics of shooting angle and distance.

From Table 1 and Figure 3, it can be seen that the common shooting strategies of different groups are determined by the players' technical preferences. For example, the probability of left shots in Group A is $43.0 \%$, and the probability of close shots and midrange shots is as high as $86.1 \%$. In subsequent matches, the opposing coach can make strategic deployments to this point to make their players pay more attention to the left side infield defense. Table 2 and Figure 4 are the analysis of players' shooting scores.

From Table 2 and Figure 4, it can be seen that there is also a big difference in the players' shooting scores. For example, the fast break scores organized by the D players have 29 points, while Group E has only 13 points, which shows the fast break of the D players. Ability is much stronger than that in Group E. In addition, players in different groups have different shooting percentages for three-pointers, two-pointers, and free throws. In this case, the coach can analyze the technical indicators of the shooting area, not only make tactical arrangements but also clearly see the number of twopointers, three-pointers, free throws, and hits of the team
TABLE 1: Statistics of shooting angle and distance.

\begin{tabular}{lcccccc}
\hline Group & $\begin{array}{c}\text { Left } \\
\text { shot }\end{array}$ & $\begin{array}{c}\text { Mid } \\
\text { shot }\end{array}$ & $\begin{array}{c}\text { Right } \\
\text { shot }\end{array}$ & $\begin{array}{c}\text { Long } \\
\text { shot }\end{array}$ & $\begin{array}{c}\text { Middle } \\
\text { distance }\end{array}$ & $\begin{array}{c}\text { Close } \\
\text { shot }\end{array}$ \\
\hline A & 43.2 & 28.5 & 28.3 & 13.9 & 39.8 & 46.3 \\
B & 35.8 & 31.7 & 32.5 & 44.8 & 36.5 & 18.7 \\
C & 19.6 & 30.2 & 50.2 & 25.5 & 50.2 & 25.3 \\
D & 48.8 & 22.3 & 28.9 & 32.3 & 34.5 & 33.2 \\
E & 19.7 & 47.6 & 32.7 & 11.2 & 35.2 & 53.6 \\
F & 28.4 & 18.2 & 53.4 & 14.2 & 45.6 & 40.2 \\
\hline
\end{tabular}

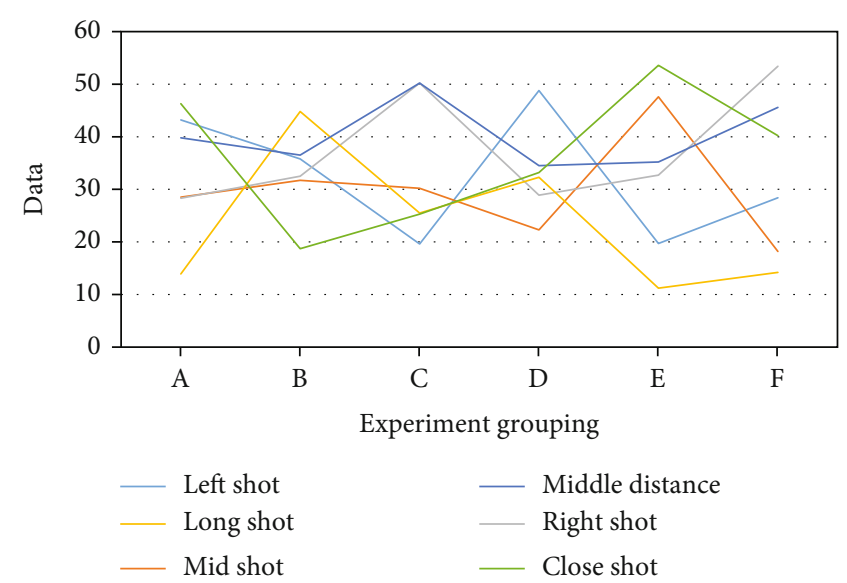

FIGURE 3: Statistics of shooting angle and distance.

TABle 2: Analysis of players' shot scores.

\begin{tabular}{lccccc}
\hline Group & $\begin{array}{c}\text { Fast break } \\
\text { score }\end{array}$ & $\begin{array}{c}\text { Second } \\
\text { attack }\end{array}$ & $\begin{array}{c}\text { Three- } \\
\text { pointer }\end{array}$ & $\begin{array}{c}\text { Two- } \\
\text { pointer }\end{array}$ & Penalty \\
\hline A & 15 & 21 & 54 & 34 & 10 \\
B & 27 & 16 & 45 & 46 & 7 \\
C & 21 & 20 & 51 & 26 & 8 \\
D & 29 & 15 & 57 & 36 & 11 \\
E & 13 & 19 & 48 & 28 & 9 \\
F & 16 & 14 & 54 & 32 & 12 \\
\hline
\end{tabular}

and each player through the data. Calculate the shooting percentage of players. Collect shooting hot zone data to make targeted defensive tactics in order to win the game. Figure 5 shows the number of dribbling rolls of the player during the dribble.

In basketball, even seemingly ordinary actions have relatively strict technical standards. For example, the most common is dribbling. Many players are prone to dribbling and turning their wrists, which leads to the violation of "carrying the ball." In the course of the experiment, this article sets a stricter index for the dribbling wrist roll, taking 90 degrees and 0 seconds as the "critical value of the $X$-axis angle of the motion sensor" and "the duration of exceeding the critical value" as the initial values. It can be seen from Figure 5 that while adjusting the parameter self-convergence, the effective monitoring ratio of the sensor is also constantly changing. From the recognition of professional coaches, 


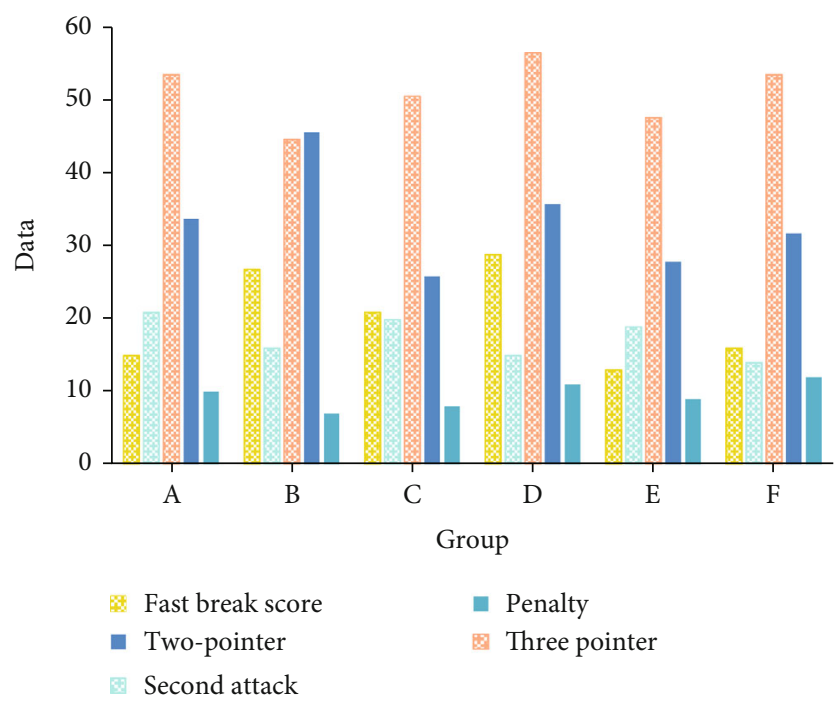

FIGURE 4: Analysis of players' shot scores.

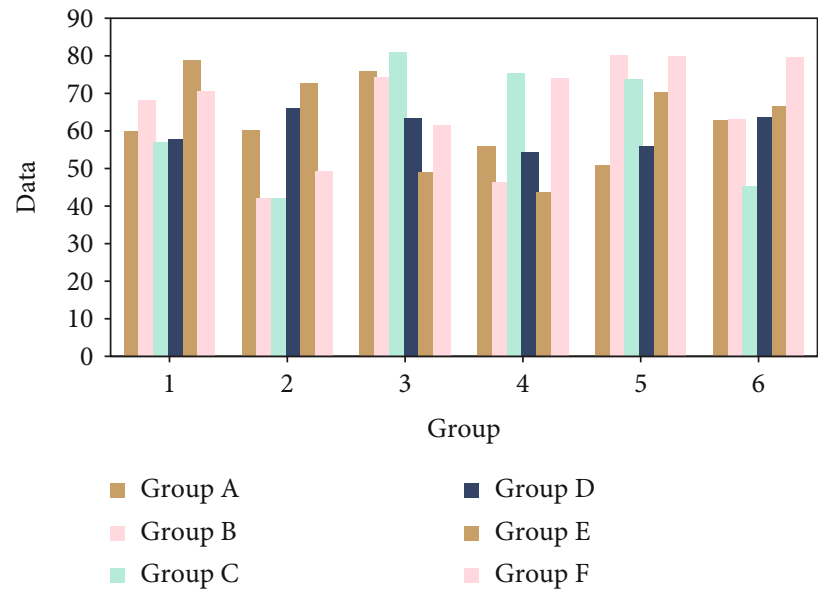

Figure 5: Number of dribbling rolls of player during the dribble.

TABLE 3: Statistics of player technical errors.

\begin{tabular}{lccccc}
\hline Group & $\begin{array}{c}\text { Dribble } \\
\text { error }\end{array}$ & $\begin{array}{c}\text { Missed } \\
\text { shot }\end{array}$ & $\begin{array}{c}\text { Rebound } \\
\text { turnover }\end{array}$ & $\begin{array}{c}\text { Pass } \\
\text { error }\end{array}$ & Foul \\
\hline A & 5 & 12 & 8 & 4 & 8 \\
B & 7 & 18 & 14 & 6 & 12 \\
C & 6 & 11 & 9 & 11 & 9 \\
D & 9 & 19 & 11 & 8 & 13 \\
E & 13 & 15 & 13 & 10 & 10 \\
F & 10 & 13 & 10 & 12 & 11 \\
\hline
\end{tabular}

when the $X$-axis angle value of the motion sensor is at a critical value of $105^{\circ}$, the system has the best effect on correcting the wrong posture of the dribble turning wrist. Table 3 and Figure 6 are the statistics of the number of players' technical errors.

Except for some minor posture errors, players may make varying degrees of errors during dribbling, shooting, rebounding, and passing. If the offensive efficiency represents the points obtained by each offensive and defensive conversion in a team game, the defensive efficiency represents the points obtained by the opponent for each offensive and defensive transition during the team competition. Offensive efficiency and defensive efficiency are added to the number of team offensive and defensive conversions, allowing this indicator to evaluate the offensive and defensive capabilities of each team based on a scientific and equal principle.

From Table 3 and Figure 6, it can be seen that Group A made fewer mistakes in dribbling and passing, but made more mistakes in shooting and rebounding. Group F had a higher number of mistakes in all aspects, which means that players need to be stronger. The coach can also set the corresponding target based on the team's average turnover rate. The coach can evaluate the performance of the team and the opponent in terms of ball control through the indicator of the turnover rate.

4.2. Motion Sensor Based on Neural Network in Physical Fitness Evaluation. For basketball players, good physical 


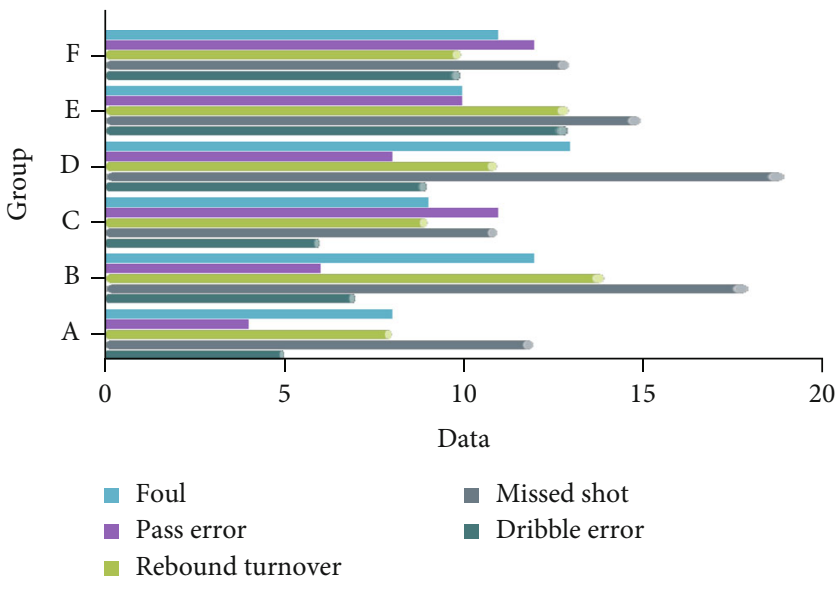

FiguRE 6: Statistics of player technical errors.

TABLE 4: Athlete's endurance test results.

\begin{tabular}{lcccr}
\hline Group & 20mSRT & 12 min & 1-mile run & $1000 \mathrm{~m}$ \\
\hline A & $63.5 \pm 14.6$ & $2413.8 \pm 192.5$ & $475.6 \pm 43.8$ & $253.3 \pm 20.8$ \\
B & $69.8 \pm 13.2$ & $2369.5 \pm 188.2$ & $486.3 \pm 45.5$ & $249.5 \pm 21.3$ \\
C & $67.7 \pm 11.6$ & $2436.7 \pm 190.3$ & $473.3 \pm 44.2$ & $251.6 \pm 22.5$ \\
D & $71.7 \pm 13.5$ & $2515.6 \pm 184.4$ & $478.5 \pm 45.8$ & $243.3 \pm 20.5$ \\
E & $72.8 \pm 14.2$ & $2452.5 \pm 193.8$ & $496.2 \pm 47.3$ & $261.7 \pm 21.9$ \\
F & $74.2 \pm 12.8$ & $2508.3 \pm 192.7$ & $458.9 \pm 42.7$ & $255.5 \pm 21.5$ \\
\hline
\end{tabular}

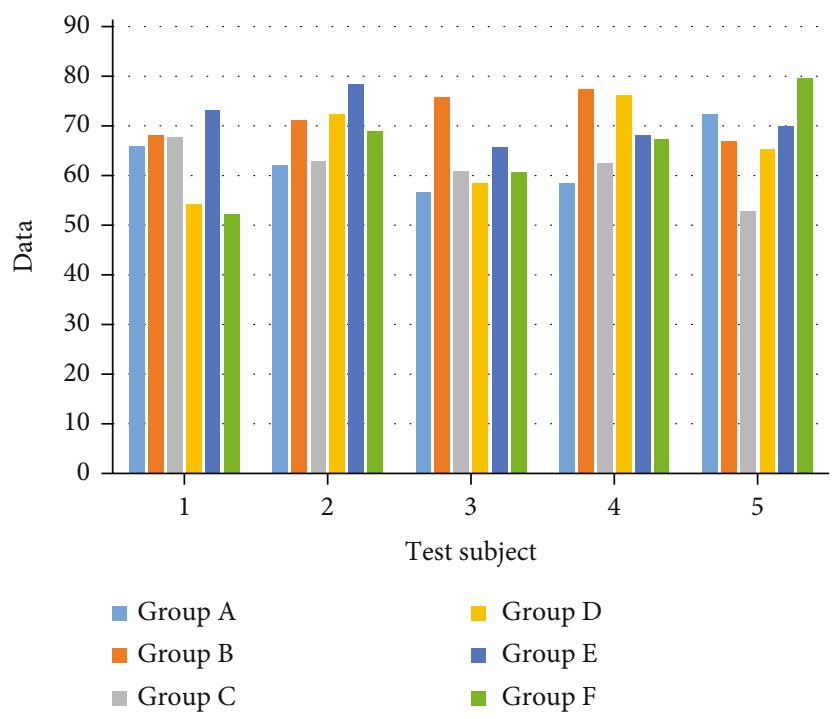

FIGURE 7: Athletes' 20-meter round-trip test data statistics.

fitness is a necessary condition for sports competition. A large amount of data information is usually involved in physical fitness assessment. These physical data not only change rapidly but also have no obvious symbolic meaning. In this case, the use of wireless sensors and intelligent analysis systems can help coaches better understand the physical fitness of athletes.
In the experiment in this article, the physical fitness of basketball players will be tested through four items: 1000meter running, 1-mile running, 20-meter round-trip running, and 12-minute long-distance running. Table 4 is the test result table of the athletes' endurance item.

It can be seen from Table 4 that the average score of the subjects in the 20-meter round-trip run was 71.95 laps, the 


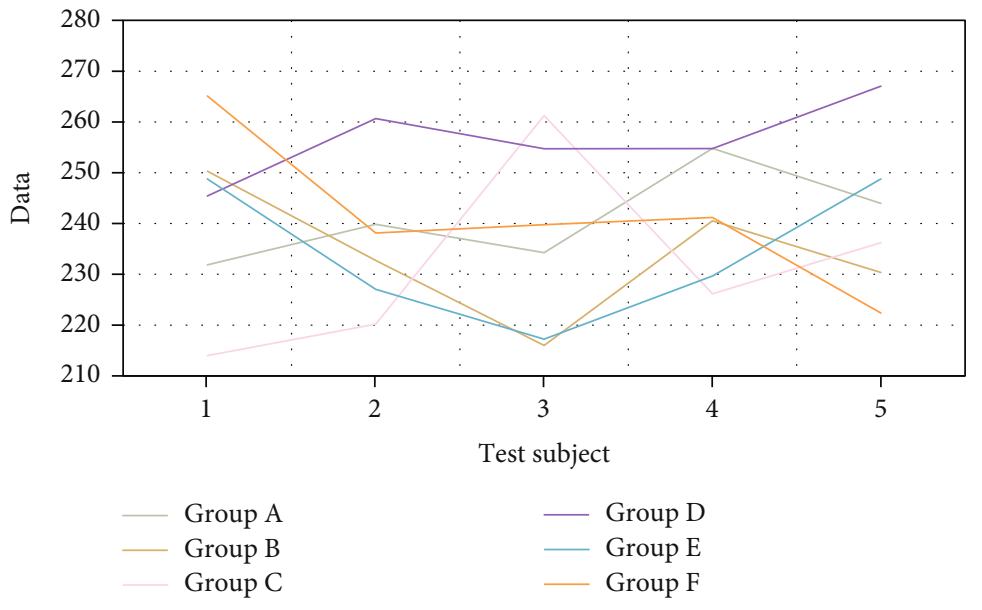

Figure 8: Athletes' 1000-meter test data.

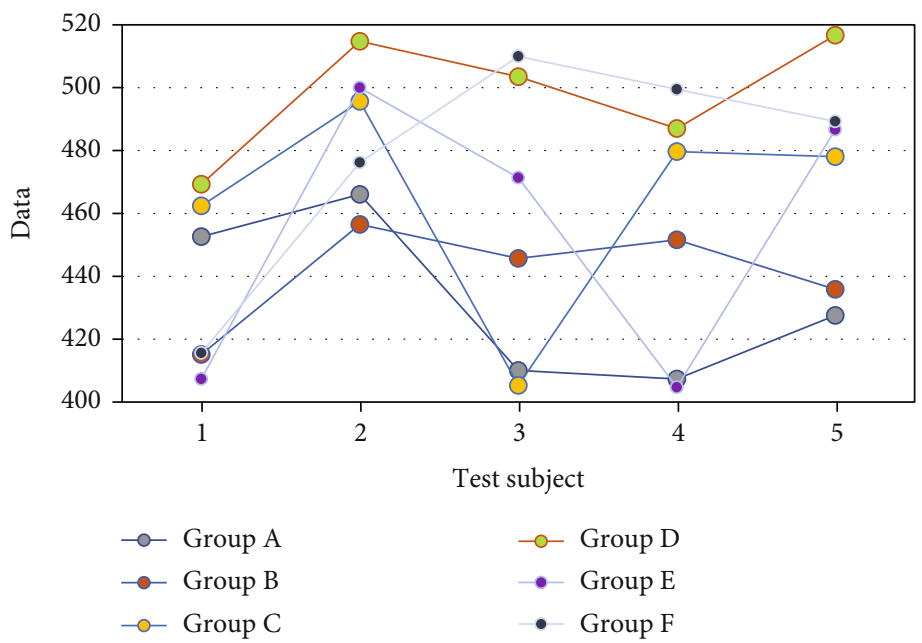

Figure 9: Athletes' 1-mile test data.

score in the 12-minute run was 2448.8 meters, the score in the 1 -mile run was 478.13 seconds, and the score in the 1000 -meter run was 252.48 seconds. Figure 7 shows the statistics of the athletes' 20-meter round-trip test data.

According to the physical fitness evaluation standard, this article believes that the data of an adult male in a 20meter round trip should reach 70 laps to be considered qualified. It can be seen from Figure 7 that only 17 of the test subjects in this article have reached the eligibility standard. Although the overall average score is 71.95 laps, there is still room for improvement in physical fitness for some people. Figure 8 is the athletes' 1000 -meter test data.

According to the physical fitness evaluation standard, this article believes that the data of adult males in the 1000meter run should be at least $272 \mathrm{~s}$ to be considered qualified. From the data in Figure 8, the average score of the 30 subjects was $252.48 \mathrm{~s}$, of which 2 did not meet the pass standard and 3 reached the excellent standard. Figure 9 is the athletes' 1-mile test data.

According to the physical fitness evaluation standard, this article believes that the qualified data for an adult male in a 1-mile run is $7: 00-8: 30(\mathrm{~min} / \mathrm{s})$. From the data in
Figure 9, the average score of the 30 subjects was $478.13 \mathrm{~s}$, of which 1 did not meet the eligibility standard and 2 reached the excellent standard.

Taken together, it can be found that most of the subjects' data in various physical fitness tests are only at the qualified level, and the number of excellent ones is still small. For basketball, endurance, explosiveness, and agility are all very important physical indicators. If you want to further improve your own basketball level, you can use the intelligent evaluation system to understand your shortcomings more clearly and perform targeted physical fitness work out.

\section{Conclusions}

In this paper, through the study of basketball technical statistical index system, according to the basketball technical statistical index system in professional competitions, the representative technical indicators are selected, and these indicators are analyzed, and their significance and function are explained. To build a complete and scientific technical statistical indicator system, "make contributions." This article conducts in-depth exploration and research on technical 
indicators such as scoring, rebounds, assists, turnovers, and hit rate and obtains effective hit rate, true hit rate, rebound efficiency, offensive and defensive conversions, offensive and defensive efficiency, assist rate, turnover rate, shooting area, scoring composition and other technical indicators which can be more intuitive, scientific, and comprehensive analysis of the substantive issues of the game. The technical indicators of rebounding are the analysis of offensive and defensive rebounding efficiency, combining the offensive and defensive conversion times of both sides to study the team's offensive and defensive efficiency; in the assist and turnover technical indicators, the offensive and defensive conversion times are added to analyze the assist efficiency and turnover efficiency.

This article uses mobile sensors and intelligent systems to evaluate the physical fitness of the subjects. The test items include four kinds, namely, 1000-meter running, 1-mile running, 20-meter round-trip running, and 12-minute longdistance running. From the test data, there is still room for improvement in the physical fitness of most subjects. Among them, the pass rate for the 1000 -meter run was $83.3 \%$, and the excellent rate was $10 \%$; the pass rate for the 1 -mile run was $90 \%$, and the excellent rate was $6.7 \%$; and the pass rate for the 20-meter round-trip run was only 56.67\%. Compared with long-distance running, round-trip running is more a test of athletes' short-term explosiveness and agility. In the follow-up exercise, subjects can start from this aspect to improve their overall physical fitness and lay the foundation for improving comprehensive basketball skills.

On the whole, the research of this article has achieved certain results. Due to the limitations of various aspects, the research still has certain deficiencies. In the future, this article suggests that the research can be improved from the following aspects: (1) starting from the changes in basketball technical indicators, further discuss the scientific nature of the technical indicators; (2) on the shooting area, assist rate, and offensive and defensive conversion times, carry out more detailed analysis; and (3) optimize intelligent algorithms to improve system data analysis and processing capabilities.

\section{Data Availability}

No data were used to support this study.

\section{Conflicts of Interest}

The authors declare that they have no conflicts of interest.

\section{Acknowledgments}

This work was supported by Jouf University, Sakaka, Al-Jouf, KSA.

\section{References}

[1] D. Conte, T. G. Favero, M. Niederhausen, L. Capranica, and A. Tessitore, "Physiological and technical demands of no dribble game drill in young basketball players," Journal of Strength \& Conditioning Research, vol. 29, no. 12, pp. 3375-3379, 2015.
[2] Y. Y. S. D. Santos, L. A. Monezi, M. S. Misuta, and L. A. Mercadante, "Technical indicators registered as a function of the playing time in Brazilian basketball," Revista Brasilra de Cineantropometria e Desempenho Humano, vol. 20, no. 2, pp. 172-181, 2018.

[3] G. Yuzhou and H. Qi, "Research on multi direction training and technical analysis of basketball based on BP neural network model," International Journal for Engineering Modelling, vol. 31, no. 1, pp. 54-60, 2018.

[4] M. Song and Y. Wang, "A study of granular computing in the agenda of growth of artificial neural networks," Granular Computing, vol. 1, no. 4, pp. 247-257, 2016.

[5] M. T. B. Florentino, E. G. da Costa, T. V. Ferreira, and A. D. Germano, "Spectral subband centroid energy vectors algorithm and artificial neural networks for acoustic emission pattern classification," Advances in Electrical and Computer Engineering, vol. 19, no. 3, pp. 49-56, 2019.

[6] T. J. Choi, J. H. Lee, H. Y. Youn, and C. W. Ahn, "Adaptive differential evolution with elite opposition-based learning and its application to training artificial neural networks," Fundamenta Informaticae, vol. 164, no. 2-3, pp. 227-242, 2019.

[7] C. H. Ko, "Accessibility of radio frequency identification technology in facilities maintenance," Journal of Engineering, Project, and Production Management, vol. 7, no. 1, pp. 45-53, 2017.

[8] W. Wang, "Digital logistics warehouse management system based on radio frequency identification technology," Science, Technology and Engineering, vol. 19, no. 2, pp. 170-174, 2019.

[9] F. Adamo, F. Attivissimo, C. Guarnieri Calo Carducci, and A. M. L. Lanzolla, "A smart sensor network for sea water quality monitoring," IEEE Sensors Journal, vol. 15, no. 5, pp. 2514$2522,2015$.

[10] M. Jaradat, M. Jarrah, A. Bousselham, Y. Jararweh, and M. al-Ayyoub, "The internet of energy: smart sensor networks and big data management for smart grid," Procedia Computer Science, vol. 56, no. 1, pp. 592-597, 2015.

[11] Z. Jiang, S. Shekhar, X. Zhou, J. Knight, and J. Corcoran, "Focal-test-based spatial decision tree learning," IEEE Transactions on Knowledge and Data Engineering, vol. 27, no. 6, pp. 1547-1559, 2015.

[12] R. Pandya and J. Pandya, "C5. 0 algorithm to improved decision tree with feature selection and reduced error pruning," International Journal of Computer Applications, vol. 117, no. 16, pp. 18-21, 2015.

[13] Y. Zhang, S. Lu, X. Zhou et al., "Comparison of machine learning methods for stationary wavelet entropy-based multiple sclerosis detection: decision tree, k-nearest neighbors, and support vector machine," SIMULATION, vol. 92, no. 9, pp. 861-871, 2016.

[14] M. Konishi, S. Okubo, T. Nishino, and M. Wakatsuki, "A decision tree analysis of a multi-player card game with imperfect information," International Journal of Software Innovation, vol. 6, no. 3, pp. 1-17, 2018.

[15] P. Rajesh, M. Karthikeyan, B. Santhosh Kumar, and M. Y. Mohamed Parvees, "Comparative study of decision tree approaches in data mining using chronic disease indicators (CDI) data," Journal of Computational and Theoretical Nanoscience, vol. 16, no. 4, pp. 1472-1477, 2019.

[16] P. Sai Diwakar Nutheti, N. Hasyagar, R. Shettar, and S. Guggari, "Ferrer diagram based partitioning technique to decision tree using genetic algorithm," International Journal 
of Mathematical Sciences and Computing, vol. 6, no. 1, pp. 2532, 2020.

[17] D. K. Chaturvedi, A. P. Sinha, and O. P. Malik, "Short term load forecast using fuzzy logic and wavelet transform integrated generalized neural network," International Journal of Electrical Power \& Energy Systems, vol. 67, pp. 230-237, 2015.

[18] E. Bassey, J. Whalley, P. Sallis, and K. Prasad, "Wavelet transform smoothing filters for metal oxide gas sensor signal cleaning," International Journal on Smart Sensing and Intelligent Systems, vol. 7, no. 5, pp. 1-5, 2020.

[19] A. A. Shah, B. S. Chowdhry, T. D. Memon, I. H. Kalwar, and J. A. Ware, "Real time identification of railway track surface faults using canny edge detector and 2D discrete wavelet transform," Annals of Emerging Technologies in Computing, vol. 4, no. 2, pp. 53-60, 2020.

[20] A. Khmag, A. R. Ramli, S. A. R. al-haddad, S. Yusoff, and N. H. Kamarudin, "Denoising of natural images through robust wavelet thresholding and genetic programming," The Visual Computer, vol. 33, no. 9, pp. 1141-1154, 2017.

[21] D. C. Jeronymo, Y. C. C. Borges, and L. . S. Coelho, "Image forgery detection by semi-automatic wavelet softthresholding with error level analysis," Expert Systems with Applications, vol. 85, pp. 348-356, 2017.

[22] X. Zhang, "A modified artificial bee colony algorithm for image denoising using parametric wavelet thresholding method," Pattern Recognition and Image Analysis, vol. 28, no. 3, pp. 557-568, 2018.

[23] P. O’Donovan, D. Coburn, E. Jones et al., "A cloud-based distributed data collection system for decentralised wastewater treatment plants," Procedia Engineering, vol. 119, no. 1, pp. 464-469, 2015.

[24] W. Yu, D. Griffith, L. Ge, S. Bhattarai, and N. Golmie, "An integrated detection system against false data injection attacks in the smart grid," Security \& Communication Networks, vol. 8, no. 2, pp. 91-109, 2015.

[25] Y. Masuda, A. Noda, and H. Shinoda, "A low power and high speed data transmission system based on 2D communication," IEICE Communications Express, vol. 5, no. 9, pp. 322-328, 2016. 